\title{
Indirect costs of depression and other mental and behavioural disorders for Australia from 2015 to 2030
}

Deborah Schofield, Michelle Cunich, Rupendra Shrestha, Robert Tanton, Lennert Veerman, Simon Kelly and Megan Passey

\section{Background}

The impact of mental disorders has been assessed in relation to longevity and quality of life; however, mental disorders also have an impact on productive life-years (PLYS).

\section{Aims}

To quantify the long-term costs of Australians aged 45-64 having lost PLYS because of mental disorders.

\section{Method}

The Survey of Disability, Ageing and Carers 2003, 2009 formed the base population of Health\&WealthMOD2030 - a microsimulation model integrating output from the Static Incomes Model, the Australian Population and Policy Simulation Model, the Treasury and the Australian Burden of Disease Study.

\section{Results}

For depression, individuals incurred a loss of AU\$1062 million in income in 2015, projected to increase to AU\$1539 million in 2030 (45\% increase). The government is projected to incur costs comprising a $22 \%$ increase in social security payments and a $45 \%$ increase in lost taxes as a result of depression through its impact on PLYS.

\section{Conclusions}

Effectiveness of mental health programmes should be judged not only in terms of healthcare use but also quality of life and economic well-being.

\section{Declaration of interest}

None.

\section{Keywords}

Mental and behavioural disorders; productive life-years (PLYS); microsimulation model.

\section{Copyright and usage}

(c) The Royal College of Psychiatrists 2019. This is an Open Access article, distributed under the terms of the Creative Commons Attribution-NonCommercial-NoDerivatives licence (http://creativecommons.org/licenses/by-nc-nd/4.0/), which permits noncommercial re-use, distribution, and reproduction in any medium, provided the original work is unaltered and is properly cited. The written permission of Cambridge University Press must be obtained for commercial re-use or in order to create a derivative work.

\section{Background}

Mental and substance-use disorders accounted for 183.9 million disability-adjusted life-years (DALYs) or 7\% of DALYs worldwide in 2010. ${ }^{1}$ Of this burden, depressive disorders accounted for the largest proportion $(41 \%)$, followed by anxiety disorders (15\%), illicit drug use (10.9\%) and alcohol use disorders (10\%). ${ }^{1}$ The global burden of mental and substance-use disorders (measured as DALYs) increased by 38\% between 1990 and 2010 as a result of population growth and ageing. ${ }^{1}$

Although burden of disease studies quantify the years and quality of life lost because of mental illness, many governments and supranational organisations (such as the Organisation for Economic Co-operation and Development (OECD)) also require, for policy development, estimates of the significant indirect costs of mental illness through non-participation in the labour market. ${ }^{2}$ The costs of mental illness through lost labour force participation and lost productivity are commonly measured as lost personal earnings. Levinson et al, using the World Health Organization's (WHO's) World Mental Health Surveys in ten high-income and nine low- and middle-income countries, found that respondents who have a serious mental illness earned, on average, a third less than the median earnings of the whole population in each country, and there were no significant between-country differences. ${ }^{3}$ These losses equated to $0.3-0.8 \%$ of national earnings. It was also found that reduced earnings among those with any earnings and the higher likelihood of not earning were both significant mechanisms for these associations. These may also result in further costs to individuals (such as falling into income poverty) and to the government (such as extra social security payments and lost tax revenue) in addition to direct (healthcare) costs. ${ }^{2}$

The direct costs are substantial as a result of the high prevalence of mental illness. ${ }^{4}$ In the USA in 2013, mental disorders surpassed all other conditions in terms of cost, with spending at \$201 billion. $^{5}$ Government spending on mental health services in Australia have increased from AU\$5.3 billion in 2004-2005 to AU $\$ 8.5$ billion in 2014-15. ${ }^{4}$ People with mental illness are also at higher risk of social and economic disadvantage. ${ }^{2,6}$

\section{Mental health impact on the labour force}

Although mental illness is more prevalent in younger age groups, older workers with a mental illness may be more likely to leave the workforce as a result of the combined effects of ageing and the disabling effects of the illness. Of the 347000 Australians aged 45-64 years out of the labour force because of ill health in 2010, $7.1 \%$ were out of the labour force as a result of depression (24 000 individuals) and another $9.6 \%$ as a result of other mental and behavioural disorders (32 000 individuals). ${ }^{6}$ Consequently, depression and other mental and behavioural disorders are ranked as the top fifth and third most common chronic health condition causing older workers to leave the labour force, respectively. ${ }^{6}$ International studies that examine the indirect costs of mental illness in adults normally focus on lost earnings for individuals ${ }^{7}$ and thus exclude other important costs such as reduced income from other sources (such as superannuation and financial assets) and retirement savings for individuals. 


\section{Study aims}

In Australia, there are two notable studies that address both the direct (medical) and indirect costs of high prevalence mental disorders (depression, anxiety-related and substance use $)^{8}$ and the low prevalent condition of psychosis ${ }^{9}$ using cross-sectional survey data. Lee et al estimated the total annual productivity losses of depression, anxiety-related and substance use to be AU\$11.8 billion, together with annual income tax losses of AU $\$ 1.2$ billion and welfare payments of AU $\$ 12.9$ billion in 2007. ${ }^{8}$ And for psychosis, Neil et al estimated the productivity costs to be AU\$40 941 and other indirect costs (for example non-government organisation assistance, supported employment and accommodation) to be AU\$14 642 per affected individual. ${ }^{9}$ However, there has been little research undertaken on quantifying the longer-term indirect costs of mental illness in Australia and internationally. This study projects the income received by people aged 45-64 who have lost productive life-years (PLYs) as a result of depression and other mental and behavioural disorders (defined as the number of people who are not in the labour force because of mental illness per year ${ }^{6}$ ), the amount of tax paid and social security payments received from 2015 to 2030. This study quantifies the difference in these costs between those who have lost PLYs as a result of depression and other mental and behavioural disorders and those in the labour force without these disorders in order to provide a more comprehensive picture of the indirect costs of mental illness than is currently available. Furthermore, these comparisons enable computation of potential savings if the illness could be prevented or treated in a way that enabled labour force participation.

\section{Method}

\section{The model}

Outputs from Health\&WealthMOD2030 - Australia's first microsimulation model of the long-term economic impact of chronic disease in people aged 45-64 - were used to quantify the impact of depression and other mental and behavioural disorders on PLYs, income, government revenue, social security payments and gross domestic product (GDP) in Australia. Information about the development of Health\&WealthMOD2030 is provided in Schofield et al. ${ }^{10}$

The base population of Health\&WealthMOD2030 was microlevel data from the Australian Bureau of Statistics' (ABS) Survey of Disability, Ageing and Carers (SDAC) in 2003 and 2009. ${ }^{11}$ Individual records were extracted for people aged 45-64 and, for each person, relevant demographic, socioeconomic and health information.

Although the SDACs 2003 and 2009 provide comprehensive data on the health and health services used by people with chronic conditions, they have only limited economic information. From another microsimulation model, Static Incomes Model (STINMOD), ${ }^{12}$ additional economic information was imputed onto the base data. STINMOD is Australia's leading model of income tax and government support payments developed by the National Centre for Social and Economic Modelling at the University of Canberra. Income, tax, social security payments and wealth data were imputed onto the base population by ascertaining individuals with similar characteristics on STINMOD and 'donating' their economic information onto Health\&WealthMOD2030 using synthetic matching. The ten variables used to perform the matching were: age group, gender, type of income unit, receipt of the Aged Pension, receipt of the Disability Support Pension (DSP), the individual's income quintile, labour force status, hours worked per week, highest educational qualification and home ownership.

Output from STINMOD was for the Australian population in 2013 and thus we indexed these data to reflect economic growth from 2013 to the projection years (2015-30). Total income and taxes paid were assumed to grow at a rate of $1 \%$ per year in real terms (i.e. $1 \%$ above the Consumer Price Index) based on information in Treasury. ${ }^{13}$ The DSP that people with mental illness may receive was benchmarked to growth in average weekly earnings. Other social security payments (including unemployment benefits or Newstart) were assumed to grow in line with the Consumer Price Index (i.e. zero real growth).

Treasury projections of population and labour force growth were used to take account of trends in these variables over the period 2015-2030. We extracted the population projections and projected full- and part-time employment rates for men and women aged $45-64$ by 5 -year age groups over 2015-30. Since Treasury's population projections were separable only by age group and gender, we applied the projected age and gender specific distributions for the remaining sociodemographic variables (education, home ownership and family structure) from a second microsimulation model, Australian Population and Policy Simulation Model, ${ }^{14}$ to project more complete profiles of the population in 2015, 2020, 2025 and 2030.

Trends in the incidence of chronic health conditions reported in Begg et $a l^{15}$ were applied to the base population. The chronic conditions trends from that study were applied from 2003 to 2023 to the base population; after this time, prevalence rates were assumed to stabilise as in Begg et al. ${ }^{15}$ The use of these data was approved by the ABS Microdata Review Panel.

\section{Lost PLYS as a result of depression and other mental health disorders}

SDAC respondents with chronic health conditions were classified by the ABS using ICD-10 codes. ${ }^{16}$ Individuals who reported their main long-term health condition as depression/mood affective disorders (excluding postpartum depression) (ICD code F30-39) were considered to have the specific condition of 'depression' in this study. Those who reported their main long-term health condition as any of the other mental and behavioural disorders i.e. dementia, schizophrenia, phobic and anxiety disorders, nervous tension/stress, intellectual and developmental disorders, intellectual disability, autism and related disorders (including Rett's syndrome and Asperger's syndrome), attention-deficit hyperactivity disorder, speech impediment, Alzheimer's disease, or other mental and behavioural disorders (not fully defined) (ICD codes F00-29 and F40-99) were considered to have 'other mental and behavioural disorders'.

In this study, those who reported they were out of the labour force because of illness and listed depression as their main longterm health condition were considered to have lost PLYs as a result of depression. Those who reported they were out of the labour force because of illness and listed another mental or behavioural disorder as their main long-term health condition were considered to have lost PLYs as a result of mental and behavioural disorders.

\section{Indirect costs}

The costs of mental illness projected over 2015-2030 were: lost income, extra social security payments and lost taxes. Personal income consisted of labour market earnings, income from other sources with a financial return and social security payments. The social security payments in STINMOD relevant for people aged 45-64 were the Aged Pension, DSP, Newstart Allowance, Carer 
Payment, and Family Tax Benefit (http://www.humanservices.gov. $\mathrm{au} /$ customer/services). The taxes paid by individuals were their personal income tax (includes Medicare levy) for the financial year. All costs were expressed in real terms in 2013 Australian dollars.

\section{Statistical analysis}

Descriptive analysis consisted of reporting the mean (s.d.) and median weekly income, social security payments and taxes paid, for individuals employed full time with no mental health condition, employed part time with no mental health condition, not in the labour force because of depression and not in the labour force because of other mental and behavioural disorders.

Differences in the costs incurred by those not in the labour force (or with lost PLY) because of depression and other mental and behavioural disorders (separately) compared with those in full- or part-time employment without these disorders were estimated using counterfactual simulation with Monte Carlo methods. For each record of those not in the labour force because of depression or other mental and behavioural disorders, a counterfactual record was selected at random with replacement from the pool of those in full-time employment without these disorders; records were matched for age group, gender and highest level of education. The mean of the difference in the costs between the records of those not in the labour force because of depression or other mental and behavioural disorders and their counterfactuals was then estimated. A total of 1000 simulations were run, generating 1000 counterfactual data-sets for records of those not in the labour force because of depression or other mental and behavioural disorders. The average of the 1000 simulations and the $95 \%$ confidence interval, estimated using the percentile method, were reported in this study.

The simulation method was repeated with the selection of counterfactual records from the pool of those in part-time employment without depression or other mental and behavioural disorders to estimate the differences in the indirect costs of those not in the labour force because of depression or other mental and behavioural disorders and those in part-time employment who do not have these disorders.

To estimate the national costs of lost labour force participation because of depression or other mental and behavioural disorders, the counterfactuals were drawn from the pool of people in the labour force (i.e. employed full time or employed part time or unemployed) without these disorders. Lost GDP per year as a result of missing workers with depression and other mental and behavioural disorders was calculated using the projected number of people out of the labour force because of these disorders from Health\&WealthMOD2030 and the projected working-age population and GDP from Treasury. All analyses were undertaken using SAS V9.4 (SAS Institute Inc., Cary, NC, USA). All statistical tests were two-sided with a $5 \%$ level of significance.

\section{Results}

Among the 5945000 people surveyed in the SDACs 2003 and 2009 who were aged 45-64 years, 27100 (1\%) were not in the labour force because of depression and a further 29900 (1\%) were not in the labour force because of other mental and behavioural disorders (supplementary Table 1; available at https://doi/org/10.1192/bjo. 2019.26). Those who were out of the labour force because of either depression or other mental and behavioural disorders had a median weekly income of AU\$421.27 in 2015. This amount of income was only $30 \%$ of the median weekly income of those employed full time without depression or other mental and behavioural disorders (AU\$1306) (supplementary Table 1). Of their total weekly income, those not in the labour force because of either depression or other mental and behavioural disorders received median social security payments of AU\$421.27 in 2015.

By 2030, the Australian population aged 45-64 years old was estimated to reach 7130200 . Of this population, there were 31200 (1\%) individuals not in the labour force because of depression and $39400(1 \%)$ not in the labour force because of other mental and behavioural disorders. Those with lost PLYs because of either depression or other mental and behavioural disorders were estimated to receive AU $\$ 484.73$ in median weekly income in 2030 (supplementary Table 1, final column). Those not in the labour force because of depression were estimated to receive median social security payments of AU\$484.73 and those not in the labour force because of other mental and behavioural disorders were estimated to receive slightly less at AU\$423.38. Being not in employment and typically with little or no other source of income, those out of the labour force because of mental illness paid a median of AU\$0 in taxes per week.

The mean differences in weekly income, social security payments and taxes between those with lost PLYs because of either depression or other mental and behavioural disorders and employed full time without these disorders (adjusted for age, gender and education) were estimated from 2015 to 2030 (Table 1). People out of the labour force because of depression received an income that was AU\$963.71 (95\% CI 876.69-1094.37) less per week than the income of those employed full time without mental disorders in 2015. Those out of the labour force because of other mental and behavioural disorders received an income that was AU\$924.81 (95\% CI 850.61-1052.28) under the weekly income of those employed full time without mental disorders in 2015.

The difference in weekly incomes between people not in the labour force because of mental illness compared with those employed full time without mental illness increased to AU\$1215.28 (95\% CI 1115.51-1372.27) for those with depression, and AU\$1171.69 (95\% CI 1083.47-1309.85) for those with other mental illnesses in 2030. People with lost labour force participation because of depression were estimated to pay AU\$336.89 (95\% CI $330.47-342.50$ ) less in taxes per week than those employed full time without mental illness in 2015, with the difference increasing to AU\$359.13 per week (95\% CI 353.83-364.26) in 2030. Similarly, those out of the labour force because of other mental and behavioural disorders paid significantly less in taxes compared with people employed full time without these disorders.

The national impact of depression when it leads to exits from the labour force was projected to be AU\$1062 million (95\% CI 953-1244 million) in lost income in 2015, increasing to AU\$1539 million (95\% CI $1387-1786$ million) in 2030 (i.e. $45 \%$ increase) (Table 2). Additional social security payments because of lost PLYs because of depression was estimated to increase from AU \$437 million (95\% CI 424-448 million) in 2015 to AU\$532 million (95\% CI 519-545 million) in 2030 - a $22 \%$ increase. Lost government tax revenue was projected to increase from AU $\$ 305$ million (95\% CI 270-345 million) in 2015 to 441 million (95\% CI $393-504$ million) in 2030 - a $45 \%$ in real terms (Table 2). Lost national income and tax revenue, and additional social security payments because of people with other mental and behavioural disorders exiting the labour force were of comparable magnitude with those for depression alone (Table 3).

As a result of the 27100 workers missing from the labour force because of depression in 2015, there was an estimated loss of AU $\$ 3.2$ billion in GDP. The number of missing workers because of depression was estimated to increase to 31200 in 2030, with the loss in GDP projected to be AU $\$ 4.3$ billion (Table 3). And as a result of the 29900 workers missing from the labour force 
Table 2 National costs of lost productive life-years because of depression and other mental and behavioural disorders per year, Australian population aged 45-64 years (in real terms, 2013 AU\$ millions)

\begin{tabular}{|c|c|c|c|c|c|c|c|c|}
\hline \multirow[b]{2}{*}{ Cost } & \multicolumn{2}{|c|}{2015} & \multicolumn{2}{|c|}{2020} & \multicolumn{2}{|c|}{2025} & \multicolumn{2}{|r|}{2030} \\
\hline & Impact & $95 \% \mathrm{Cl}$ & Impact & $95 \% \mathrm{Cl}$ & Impact & $95 \% \mathrm{Cl}$ & Impact & $95 \% \mathrm{Cl}$ \\
\hline \multicolumn{9}{|l|}{ Depression } \\
\hline Lost income & 1062 & (953 to 1244$)$ & 1243 & (1114 to 1424$)$ & 1391 & (1254 to 1579$)$ & 1539 & (1387 to 1786) \\
\hline Extra social security payments & 437 & (424 to 448 ) & 479 & (467 to 491$)$ & 499 & (487 to 511$)$ & 532 & (519 to 545 ) \\
\hline Lost income tax revenue & 305 & (270 to 345$)$ & 361 & (321 to 406) & 402 & (358 to 454 ) & 441 & (393 to 504) \\
\hline \multicolumn{9}{|c|}{ Other mental and behavioural disorders } \\
\hline Lost income & 1109 & (997 to 1282 ) & 1273 & (1149 to 1470$)$ & 1518 & (1377 to 1750$)$ & 1876 & (1698 to 2126) \\
\hline Extra social security payments & 432 & (418 to 445 ) & 472 & (460 to 472 ) & 528 & (515 to 542$)$ & 630 & (617 to 630) \\
\hline Lost income tax revenue & 308 & (276 to 351$)$ & 357 & (357 to 405) & 426 & (382 to 481$)$ & 532 & (473 to 594 ) \\
\hline
\end{tabular}

because of other mental and behavioural disorders, there was an estimated loss of AU\$3.5 billion in GDP in 2015. The number of missing workers because of other mental and behavioural disorders increased to 39400 in 2030 and the associated loss in GDP was projected to be AU $\$ 5.5$ billion.

\section{Discussion}

\section{Main findings}

This study projected the national costs of depression and other mental and behavioural disorders through lost PLYs among people aged 45-64 years to increase over the next 15 years. For depression, these costs consisted of a $45 \%$ increase in lost income (which grew faster than the $22 \%$ growth in social security payments as a result of the indexation of these payments being less than expected wages growth), a $45 \%$ increase in lost income tax revenue, and a 34\% increase in lost GDP from 2015 to 2030.

These indirect costs are in addition to the direct costs of mental illness. The direct (healthcare) cost of mental illness was estimated to be AU $\$ 8.5$ billion for all age groups in 2014-15, which covered mental health spending in hospitals, out-of-hospital, pharmaceuticals and community services. ${ }^{4}$ However, it should be noted that only $62 \%$ of people with mental illness actually seek help from healthcare professionals/medical facilities in Australia ${ }^{17}$ and thus the direct costs would be greater if there were sufficient services available to meet that higher demand. Significant other spending also occurs for housing and accommodation, employment and disability services for those with mental illness. ${ }^{8,9}$

\section{Limitations}

This study does have some limitations. First, the findings are derived from respondents' self-reported labour market behaviour and whether they have mental illness or not. Despite self-reported employment status and health in general being considered as valid measures for these types of studies, ${ }^{18}$ there is the potential for bias in results. Second, the findings on the indirect costs of mental illness presented in this paper are for a single country (Australia). However, we also make the point that these findings demonstrate how microsimulation modelling can be used to obtain estimates of the number of people in a population effected by mental illness and their (and the government's) costs and that concerns about the direct and indirect costs of mental illness are relevant to OECD countries. ${ }^{2}$

\section{Comparison with findings from other studies}

Other studies have estimated the costs of lost labour force participation as a result of mental illness as the number of lost work days, lost earnings or the amount of disability support payments. ${ }^{7-9,19}$ However, these studies are limited in scope compared with the current study; for example, they did not include all (and count specific) social security payments and lost GDP. They have a number of other limitations, including only using average earnings to estimate the cost to individuals or average of total support payments to estimate the cost to government. Such average measures may not be representative of the actual economic circumstances of the population with mental illness. Our study used individual-level income, taxes paid and social security payments to estimate the costs to individuals from exiting the labour market as a result of either depression or other mental and behavioural disorders. The study by Lee et $a l^{8}$ reports similar estimates of the costs of lost productivity and lost taxes for mental illness when considering the entire group (i.e. those with depression and other behavioural disorders). However, there is a notable difference in the estimates of the cost of welfare payments between these studies, which is likely to stem from the lack of detailed information about the type of pensions and allowances received by individuals that comprises the income of this group (and hence the use of an average value of welfare benefits) in Lee et al. ${ }^{8}$ Finally, the previous studies (with the exception of McCrone $e t a l^{7}$ ) did not use a study design (such as microsimulation) enabling estimation of the long-term costs of mental illness.

\section{Implications}

Despite the significant increase in government spending on mental health services over the past 25 years, ${ }^{4,8,9}$ the costs are continuing to surge. This circumstance adds credence to the government expanding its investment in prevention as well as maintaining support strategies for mental illness. The government is likely to further benefit from these strategies through the reduction in adverse health, family, and work consequences (such as preventing the loss of income tax and payroll tax); reduced social security payments; and reduced spending on other services (such as healthcare, education, housing and the justice system).

\begin{tabular}{|c|c|c|c|c|c|c|c|c|}
\hline \multirow[b]{2}{*}{ Mental illness } & \multicolumn{2}{|c|}{2015} & \multicolumn{2}{|c|}{2020} & \multicolumn{2}{|c|}{2025} & \multicolumn{2}{|c|}{2030} \\
\hline & $\begin{array}{l}\text { Lost GDP, } \\
\text { \$AU billions }\end{array}$ & $\begin{array}{c}\% \text { of } \\
\text { total GDP }\end{array}$ & $\begin{array}{l}\text { Lost GDP, } \\
\text { \$AU billions }\end{array}$ & $\begin{array}{c}\% \text { of } \\
\text { total GDP }\end{array}$ & $\begin{array}{l}\text { Lost GDP, } \\
\text { \$AU billions }\end{array}$ & $\begin{array}{c}\% \text { of } \\
\text { total GDP }\end{array}$ & $\begin{array}{l}\text { Lost GDP, } \\
\text { \$AU billions }\end{array}$ & $\begin{array}{c}\% \text { of } \\
\text { total GDP }\end{array}$ \\
\hline Depression & 3.17 & 0.21 & 3.55 & 0.21 & 3.88 & 0.20 & 4.33 & 0.20 \\
\hline Other mental and behavioural disorders & 3.50 & 0.24 & 3.90 & 0.23 & 4.50 & 0.24 & 5.47 & 0.25 \\
\hline
\end{tabular}


Despite strong economic growth and a low national unemployment rate, Australia has some of the lowest employment rates for people with mental illness among the OECD. For example, the employment gap between people who have mental health problems and those who do not in Australia is about 20 percentage points - a gap wider than in any of the other eight OECD countries involved in the OECD's ninth and last review of what these countries are doing to tackle the broader health, education, social and labour market policy challenges in relation to mental illness among the workingage population. ${ }^{20}$ Australians effected by mental illness are also three times more likely to be unemployed than those who have no mental health problems and are overrepresented in all benefit schemes. Even when they do have jobs, people with mental illness often struggle with more and longer periods of sickness absence and underperformance at work. ${ }^{20}$ Thus Australia's labour market and related structures may not be adequately supporting the employment of people with mental illness.

For these reasons, the Australian Federal Government has put forward a multifaceted strategy with the main goals being: the prevention and early detection of mental illness; greater assistance for individuals to better understand and manage their mental illness; the elimination of discrimination, and assistance for employers and work colleagues in becoming more aware of the value of workers with mental illness; the government helping where necessary with the costs of modifying workplaces and enhancing access to education and training; and helping individuals with mental illness to remain connected with society. ${ }^{21}$ Since the strategy involves the government working with a range of stakeholders and institutions, a cross-portfolio approach is necessary in order to achieve improvements in mental health outcomes and generate the kind of opportunities needed for people to experience 'a contributing life' that includes employment, housing and social connectedness. ${ }^{21}$ A similar approach has been adopted in other OECD countries; for example, the UK has been operating a 'cross-government' mental health outcomes plan called 'No health without mental health' since $2011 .^{22}$

\section{Interventions}

A recent systematic meta-review assessing the effectiveness of workplace interventions for the prevention, treatment or rehabilitation of workers diagnosed with depression, anxiety or both mental illnesses $^{23}$ found that the quality of evidence was moderate for two primary prevention interventions: enhancing the worker's control, and encouraging physical activity. Higher-quality evidence was found for cognitive-behavioural therapy (CBT)-based stress management; however, poorer-quality evidence was found for other secondary prevention interventions (such as counselling). Tertiary interventions with a particular focus on improving labour force participation or the nature of the individual's employment status (such as exposure therapy and CBT-based and problem-focused return to work interventions) generated high-quality evidence for improving the symptoms of depression, anxiety or both illnesses and moderate evidence for improving occupational outcomes. In short, this review demonstrated that there is reliable empirical evidence for the effectiveness of interventions implemented in the workplace that could be used more widely to help in the prevention of depression, anxiety or both illnesses, as well as assisting with the recovery of workers diagnosed with these illnesses.

The costs of mental illness to individuals are not only sizeable but potentially perpetuating. The lower labour force participation rate for people with mental illness means that they are also more susceptible to falling into poverty not only in their prime working-age years but also in retirement as a result of lack of accumulated savings. Consequently, mental illness can generate a lifetime of social and economic marginalisation. ${ }^{22}$ Thus assisting people with mental illness to participate in the labour force through effective workplace interventions (such as those detailed in Joyce $e a^{23}$ ), along with the government taking a cross-portfolio approach to assist people with mental illness across various aspects of their lives (such as housing, education and work), will not only help them to achieve greater economic well-being, but also enable them to experience greater social connectedness with additional positive mental health outcomes.

\section{Scaling-up government initiatives to tackle mental illness}

It is important to note that the significant costs of depression and other mental and behavioural disorders are incurred in low-, medium- and high-income countries. A recent study has quantified not only the direct (medical treatment) costs and health outcomes but also some of the indirect costs attributable to depression and anxiety disorders in 36 low-, middle- and high-income countries from 2016 to $2030 .^{24}$ The estimated costs of scaling-up effective medical treatment coverage for depression and anxiety disorders (mainly counselling and antidepressant medication) totalled US $\$ 147$ billion. However, the benefits of this investment were shown to significantly outweigh these treatment costs. For instance, a 5\% improvement in labour force participation and productivity was estimated to be worth US\$399 billion, and improvements in health (measured as healthy life-years) contributed a further US $\$ 310$ billion in benefits. ${ }^{24}$ However, these type of large-scale benefits will only be possible if the level of national investment in mental health services is increased to match the level of national need. The WHO's Mental Health Atlas Survey for 2014 suggests that governments are currently spending, on average, $3 \%$ of their total health budgets on mental health, with allocations ranging from less than $1 \%$ in low-income countries to $5 \%$ in high-income countries. ${ }^{25}$ In order to reduce the significant societal costs of depression and other mental and behavioural disorders projected for low, medium- and high-income countries if current investment levels remain, both the WHO and the World Bank have commenced initiatives to assist countries with scaling-up their investments placing investment in effective treatment for mental disorders at the centre of the global development agenda. ${ }^{26}$

From a global perspective, it would also be informative to know if similar results occurred in other OECD nations or developing nations. With growth in the development and use of microsimulation models across the world, these issues may be the focus of future research that may support and generalise the results of the current study even further.

Deborah Schofield, PhD, Chair and Professor of Health Economics, Director, Centre for Ecoran schofield, PhD, Chair and Professor of Health Economics, Director, Centre for of Business and Economics, Macquarie University, Australia; Michelle Cunich, PhD, Research Fellow in Health Economics, The Boden Institute of Obesity, Nutrition, Exercise and Eating Disorders; and Director, Sydney Health Economics, Sydney Local Health District, Charles Perkins Centre, The University of Sydney, Australia;

Rupendra Shrestha, PhD, Senior Research Fellow, Health Economics, Faculty of Pharmacy, The University of Sydney; and Centre for Economic Impacts of Genomic Medicine (GENImpact), Department of Economics, Faculty of Business and Economics, Macquarie University, Australia; Robert Tanton, PhD, Professor, National Centre for Social and Economic Modelling, University of Canberra, Australia; Lennert Veerman (1D), PhD, Senior Health Economist, Cancer Council NSW; and Professor of Public Health, School of Medicine, Griffith University, Gold Coast campus, Australia; Simon Kelly, PhD, Professor, National Centre for Social and Economic Modelling, University of Canberra, Australia; Megan Passey, PhD, Associate Professor, University Centre for Rural Health, School of Public Health, The University of Sydney, Australia

Correspondence: Michelle Cunich, The Boden Institute of Obesity, Nutrition,

Exercise and Eating Disorders, Sydney Health Economics, Sydney Local Health District, D17 - Charles Perkins Centre, The University of Sydney, NSW 2006, Australia. E-mail: michelle.cunich@sydney.edu.au

First received 8 Jan 2018, final revision 3 Mar 2019, accepted 29 Mar 2019 


\section{Funding}

The development of the microsimulation model used in this study, Health\&WealthMOD2030, is funded by the Australian Research Council (under grant LP100100158) and Pfizer Australia is a partner to the grant. M.P. is funded by Fellowships from the National Health and Medical Research Council of Australia and the Sydney Medical Foundation. All authors are independent from the funding sources, and the funding sources (including Pfizer Australia) played no part in the research design, undertaking of the analysis, formulation or interpretation of the results, decision to publish the research findings, nor any other part of the research process.

\section{Supplementary material}

Supplementary material is available online at https://doi.org/10.1192/bjo.2019.26.

\section{References}

1 Whiteford HA, Degenhardt L, Rehm J, Baxter AJ, Ferrari AJ, Erskine HE, et al. Global burden of disease attributable to mental and substance use disorders: findings from the Global Burden of Disease Study 2010. Lancet 2013; 382: 1575-86.

2 OECD. Making Mental Health Count: The Social and Economic Costs of Neglecting Mental Health Care, OECD Health Policy Studies. OECD Publishing, 2014.

3 Levinson $\mathrm{D}$, Lakoma $\mathrm{M}$, Petukhova $\mathrm{M}$, Schoenbaum $\mathrm{M}$, Zaslavsky $\mathrm{A}$ Angermeyer $\mathrm{M}$, et al. Associations of serious mental illness with earnings: results from the WHO World Mental Health surveys. Br J Psychiatry 2010; 197: 114-21.

4 Australian Institute of Health and Welfare. Expenditure on Mental Health Services. AlHW, 2016.

5 Roehrig C. Mental disorders top the list of the most costly conditions in the United States: \$201 billion. Health Aff (Millwood) 2016; 35:1130-5.

6 Schofield D, Shrestha R, Cunich M, Tanton R, Kelly S, Veerman L, et al. Lost productive life years caused by chronic conditions in Australians aged 45-64 years, 2010-2030. Med J Aust 2015; 203: 260.e1-6.

7 McCrone P, Dhanasiri S, Patel A, Knapp M, Lawton-Smith S. Paying the Price: The Cost of Mental Health Care in England to 2026. Kings Funds, 2008.

8 Lee Y-C, Chatterton ML, Magnus A, Mohebbi M, Le LKD, Mihalopoulos C. Cost of high prevalence mental disorders: findings from the 2007 Australian National Survey of Mental Health and Wellbeing. Aust N Z J Psychiatry 2017; 51: 1198-211.

9 Neil AL, Carr VJ, Mihalopoulos C, Andrew Mackinnon A, Morgan VA. Costs of psychosis in 2010: findings from the second Australian National Survey of Psychosis. Aust N Z J Psychiatry 2014; 48: 169-82.

10 Schofield D, Shrestha R, Kelly S, Veerman L, Tanton R, Passey $M$, et al Health\&WealthMOD2030: a microsimulation model of the long term economic

impacts of disease on the labour force participation of Australians aged 45-64 years old. Int J Microsimulation 2014; 7: 94-118.

11 Australian Bureau of Statistics. Information Paper - Basic Confidentialised Unit Record File: Survey of Disability, Ageing and Carers 2003 (reissue). Australian Bureau of Statistics, 2005

12 Percival R, Abello A, Vu QN. STINMOD (Static Income Model). In Modelling Our Future: Population Ageing, Health and Aged Care (eds A Gupta, A Harding). Elsevier BV, 2007.

13 Treasury. Budget Paper No 1: Budget Strategy and Outlook 2015-16. Commonwealth Treasury, 2015.

14 Keegan M, Kelly S. APPSIM - Dynamic Microsimulation Modelling of Social Security and Taxation. National Centre for Social and Economic Modelling University of Canberra, 2009.

15 Begg SJ, Vos T, Barker B, Stanley L, Lopez AD. Burden of disease and injury in Australia in the new millennium: measuring health loss from diseases, injuries and risk factors. Med J Aust 2008; 188: 36-40.

16 World Health Organization. The ICD-10 Classification of Mental and Behavioural Disorders: Clinical Descriptions and Diagnostic Guidelines. WHO, 1992.

17 Andrews G, Hall W, Teesson M, Henderson S. The Mental Health of Australians. Commonwealth Department of Health and Aged Care, 1999.

18 Cai L, Kalb G. Health Status and Labour Force Participation: Evidence from the HILDA Data. Melbourne Institute of Applied Economic and Social Research, 2004

19 Goetzel RZ, Hawkins K, Ozminkowski RJ, Wang S. The health and productivity cost burden of the top 10 physical and mental health conditions affecting six large U.S. employers in 1999. J Occup Environ Med 2003; 45: 5-14.

20 OECD. Sick on the Job? Myths and Realities about Mental Health and Work. OECD Publishing, 2012.

21 National Mental Health Commission. Contributing Lives, Thriving Communities: Report of the National Review of Mental Health Programmes and Services. National Mental Health Commission, 2014.

22 HM Government. No Health Without Mental Health: A Cross-Government Mental Health Outcomes Strategy for People of All Ages. Crown, 2011.

23 Joyce $S$, Modini $M$, Christensen $H$, Mykletun A, Bryant R, Mitchell $P$, et al. Workplace interventions for common mental disorders: a systematic metareview. Psychol Med 2016; 46: 683-97.

24 Chisholm D, Sweeny $K$, Sheehan $P$, Rasmussen B, Smit F, Cuijpers $P$, et al. Scaling-up treatment of depression and anxiety: a global return on investment analysis. Lancet Psychiatry 2016; 3: 415-24.

25 World Health Organization. Mental Health Atlas 2014. WHO, 2015.

26 The World Bank. Mental Health (Brief). The World Bank, 2017. (http://www. worldbank.org/en/topic/health/brief/mental-health) 